\title{
Total knee arthroplasty in the valgus knee
}

\author{
Roberto Rossi • Federica Rosso • Umberto Cottino • \\ Federico Dettoni • Davide Edoardo Bonasia • \\ Matteo Bruzzone
}

Received: 17 November 2013 / Accepted: 24 November 2013/Published online: 24 December 2013

(C) Springer-Verlag Berlin Heidelberg 2013

\begin{abstract}
Valgus knee deformity is a challenge in total knee arthroplasty (TKA) and it is observed in nearly $10 \%$ of patients undergoing TKA. The valgus deformity is sustained by anatomical variations divided into bone remodelling and soft tissue contraction/elongation. Bone tissue variations consist of lateral cartilage erosion, lateral condylar hypoplasia and metaphyseal femur and tibial plateau remodelling. Soft tissue variations are represented by tightening of lateral structures: lateral collateral ligament, posterolateral capsule, popliteus tendon, hamstring tendons, the lateral head of the gastrocnemius and iliotibial band. Complete pre-operative planning and clinical examination are mandatory to manage bone deformities and soft tissue contractions/elongations and to decide if a higher constrained prosthesis is necessary. Two different approaches have been described to perform TKA in a valgus knee: the anteromedial approach and the anterolateral one. In valgus knee deformity bone cuts can be performed differently in order to correct low-grade deformities and reduce great deformities. There is still debate in the literature on the sequence of lateral soft tissue release to achieve the best alignment without any instability. The aim of this article is to review the anatomical variations underlying a valgus knee,
\end{abstract}

\author{
R. Rossi $(\bowtie) \cdot$ F. Dettoni $\cdot$ M. Bruzzone \\ AO Mauriziano Umberto I, Department of Orthopedics and \\ Traumatology, Largo Turati 62, 10129 Turin, Italy \\ e-mail: rossir@fastwebnet.it \\ F. Rosso $\cdot$ U. Cottino \\ University of Study of Turin, Via Giuseppe Verdi, 8, 10100 Turin, \\ Italy \\ D. E. Bonasia \\ AO CTO-M. Adelaide, Department of Orthopedics and \\ Traumatology, Via Zuretti 29, 10126 Turin, Italy \\ R. Rossi \\ SCDU Ortopedia e Traumatologia, AO Mauriziano Umberto 1, \\ Largo Filippo Turati 62, 10128 Turin, Italy
}

to assess the best pre-operative planning and to evaluate how to choose the grade of constraint of the implant. We will also review the main approaches and surgical techniques both for bone cuts and soft tissue management. Finally, we will report on our experience and technique.

Keywords Knee $\cdot$ Valgus $\cdot$ Arthroplasty

\section{Introduction}

Valgus knee deformity is a challenge in total knee arthroplasty (TKA). This deformity (defined as a valgus angle equal to or greater than $10^{\circ}$ ) is observed in nearly $10 \%$ of patients undergoing TKA [1]. It can be congenital or secondary to osteoarthrosis, rheumatic diseases, post-traumatic arthritis and to an over-correction consequent to a valgus osteotomy [2]. Excessive pre-operative malalignment predisposes to a greater risk of failure compared to well-aligned knees. For this reason it is important to correct the deformity during surgery even if it does not completely eliminate the increased risk of failure (1.9 vs $0.5 \%$ ) [3]. The valgus deformity is sustained by anatomical variations divided into bone tissue remodelling and soft tissue contraction/elongation. Bone tissue variations consist of lateral cartilage erosion, lateral condylar hypoplasia and metaphyseal femur and tibial plateau remodelling. Soft tissue variations are represented by tightening of lateral structures: lateral collateral ligament (LCL), posterolateral capsule (PLC), popliteus tendon (POP), hamstring tendons, the lateral head of the gastrocnemius (LHG) and iliotibial band (ITB). Some authors also described a posterior cruciate ligament (PCL) alteration in valgus knees, but in the literature its influence in maintaining the deformity is not universally recognised. The described deformities can lead to a tibial external rotation and to a patellar lateral subluxation tendency [4]. Three grades of valgus deformity have been described (I, 
II, III) $[1,5]$. In grade I the deviation is less than $10^{\circ}$, passively correctable, with contracture of the lateral soft tissue but without elongation of the medial collateral ligament (MCL, $80 \%$ of cases). In grade II the axial deviation ranges between 10 and $20^{\circ}$, the lateral structures are contracted and the MCL is elongated but functional ( $15 \%$ of cases). Grade III deformity is present in the remaining $5 \%$ of the patients; the axial deformity is greater than $20^{\circ}$, the lateral structures are tight and the medial stabilisers are not functional. Valgus deformity, for these reasons, is a challenge for the surgeon both for gap balancing and implant constraint choice. Several different surgical techniques have been described to perform TKA in valgus deformity; the aim of this article is to give an overview of the most common approaches and to present our choice.

\section{Pre-operative planning and implant selection}

\section{(a) Radiographic planning}

In our experience mandatory pre-operative radiographs of the knee undergoing TKA are: weightbearing anteroposterior, lateral, Rosenberg and Merchant views. These are useful for planning and bone stock evaluation. Attention should be focused on lateral distal femoral hypoplasia, posterior femoral condyle erosion and metaphyseal remodelling both of the femur and tibia, which can lead to malalignment or malrotation of the femoral component. The patellofemoral joint can be partially dislocated. In addition anteroposterior and lateral views are mandatory to evaluate the amount of osseous resection needed to correct deformities without leading to knee instability. A weight-bearing long leg view is fundamental for the evaluation of lower limb alignment, deformity level and to plan the amount of correction $[1,6]$.

(b) Knee evaluation

The overall alignment should be assessed both in the supine and weight-bearing positions, and the gait should be observed, in order to identify other dynamic instabilities. Any sagittal deformity, such as fixed flexion contracture or recurvatum, as well as any rotational deformity, should be tested during the physical examination. The knee should be evaluated for anteroposterior laxity, range of motion (ROM), coronal and sagittal deformity, and mediolateral instability. A crucial point is to assess if valgus deformity is fixed or still reducible. If a fixed deformity is present, the lateral structure is really tight and medial ligaments are partially continent. In these cases after the lateral soft tissue release, there may be a laxity requiring the use of a constrained prosthesis. If the deformity is reducible, soft tissue release will be less invasive, and a standard unconstrained prosthesis could be sufficient [6]. Figure 1 shows a reducible valgus knee. (c) Templating

In the radiographic anteroposterior view of the knee, a template of bone cuts should be performed. A line is drawn on the tibial anatomical axis and then a perpendicular one is drawn at the level of the lateral tibial plateau. This will give the surgeon an indication for the tibial resection. The femoral anatomical axis is drawn and then a second line with the desired amount of valgus (usually $3^{\circ}$ ) is also drawn at the level of the intercondylar notch. Figure 2 shows this kind of planning.

On the lateral radiograph the surgeon should investigate the presence of osteophytes in the posterior capsule; furthermore, a lateral view can be used for sizing the femoral component [1] and for locating the entry point of the femoral canal.

(d) Selection of the implant

The implant selection should be carried out pre-operatively, based on the radiological and clinical evaluation.

There is an open debate in the literature between posterior-stabilised (PS) and cruciate-retaining (CR) implants, also in valgus deformity. In valgus deformity, the PCL is often contracted and it may limit the deformity correction [7]. Furthermore, it may be more difficult to obtain the deformity correction with an intact PCL, since the PCL is a secondary stabiliser $[8,9]$. Besides, the PS design is more stable than a CR one because of the postcam mechanism and the PS design allows for greater lateralisation of the femoral and tibial components, which improves patellar tracking and minimises the necessity to perform a lateral retinacular release [1]. For these reasons some authors suggested that it is simpler to substitute a contracted PCL with a PS design than to stabilise it using a CR implant, recommending that a PS design be used in valgus deformity [2]. Figures 3 and 4 show a case in which a PS implant has been used in a valgus deformity. There is some concern about the association between planovalgus foot and failure of a CR implant. As described in the literature, a careful foot examination is mandatory to check for the presence of ipsilateral posterior tibial tendon insufficiency, especially in patients with a valgus knee [10]. Figure 5 shows a case of valgus knee associated with a planovalgus foot.

Another debated issue is the amount of constraint needed to balance a valgus knee. Careful pre-operative planning is essential to estimate the grade of constraint that will be necessary during surgery. Accurate physical examination is mandatory to evaluate if a valgus deformity is fixed or reducible: if a fixed valgus deformity is present, after lateral soft tissue release the knee may be unstable because both the medial and lateral structures will no longer be functional. In these cases the surgeon should plan to also have a semi-constrained prosthesis in the operating theatre, such as a constrained condylar one. 
Fig. 1 Clinical examination showing a reducible valgus knee. a Valgus knee. b Reduction of the valgus during a varus stress

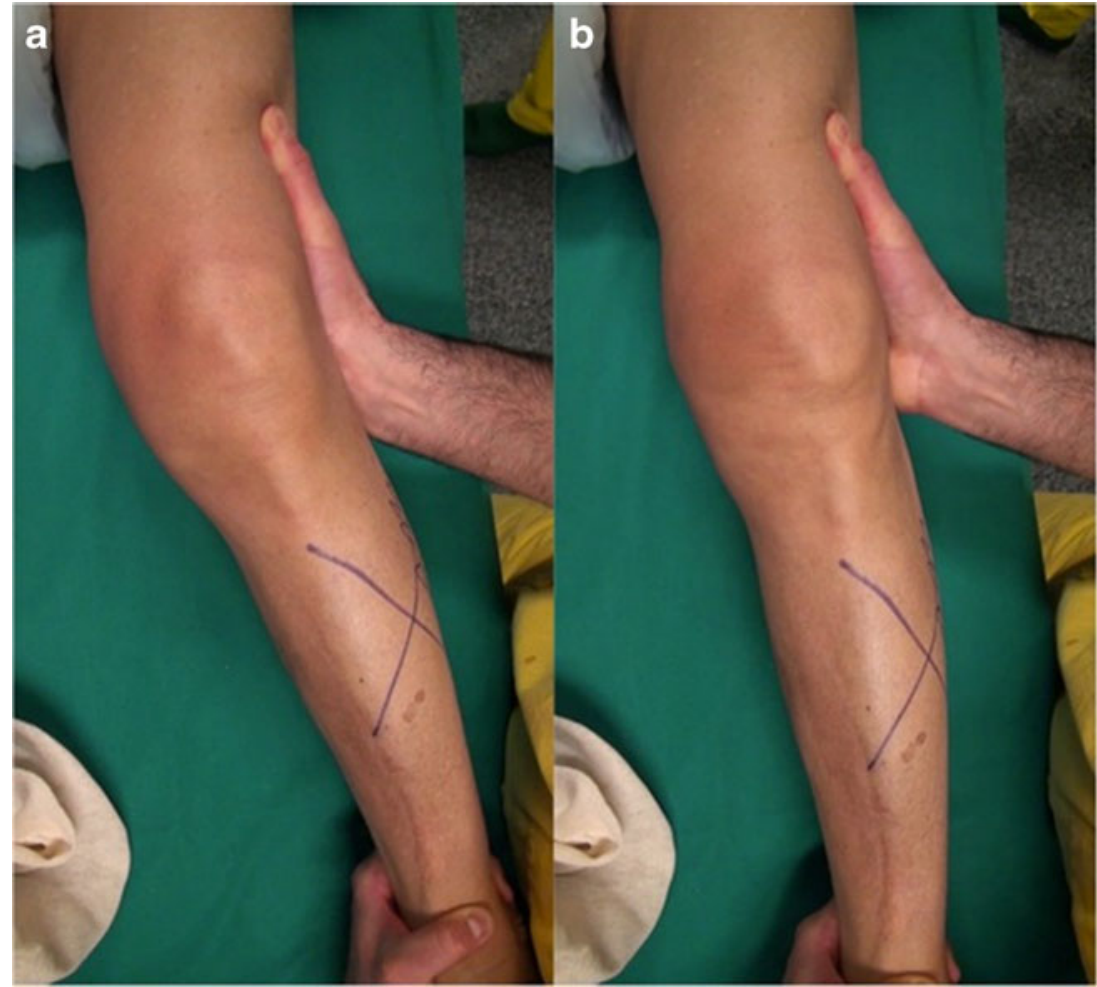

There is some agreement in the literature that the surgeon should resist the temptation to use a higher constrained prosthesis. In 2013 [11] Pang et al. reported a retrospective review of patients with a type II valgus knee who underwent primary TKA. There were 50 patients in whom a constrained TKA was performed and another 50 cases who received a PS or CR prosthesis. They concluded that there were no statistically significant differences in anteroposterior and mediolateral stability between the two groups. Constrained TKA were associated with more significant joint line changes compared with the unconstrained ones.

On the other hand, in grade III valgus deformity, in which the medial soft tissue is no longer functional with certainty, a higher constrained prosthesis is mandatory to achieve a stable knee [1]. Figures 6 and 7 show a case of grade III valgus knee in which a higher constrained prosthesis was implanted.

(e) Osteotomy in the valgus knee and TKA

Patients with genu valgum and isolated lateral compartment osteoarthritis are candidates for distal femoral varus osteotomy.

Osteotomies are performed in order to delay TKA implant [12] and to correct extra-articular deformities leading to early arthritis [13]. When the implant of a TKA cannot be delayed anymore, it can be more challenging if an osteotomy has been previously performed. The results of these procedures still remain controversial in the literature [13,14]. In the past, if arthritis and extra-articular deformity coexisted, simultaneous corrective osteotomy of angular deformity and TKA have been considered the correct treatment. This highly demanding procedure increases the risk of poor TKA result in the case of osteotomy failure. It is important to consider that even in extra-articular deformities alignment correction is possible in the majority of cases without extra-articular procedures. Good pre-operative planning and templating, intraarticular bone resection and soft tissue balancing in flexion and extension are necessary $[15,16]$.

\section{Approaches}

Anteromedial approach

The anteromedial approach is used by the majority of authors and there are no contraindications even in the valgus knee [1, 5]. The patellar dislocation is usually easy with this approach, because of the combination of the valgus deformity and the lateralisation of the tibial tuberosity (TT) [2]. When a medial approach is used in a valgus knee, the surgeon should be very careful with the MCL detachment: the release of the medial structures should be minimised in the valgus deformities and limited to overhanging osteophytes. 


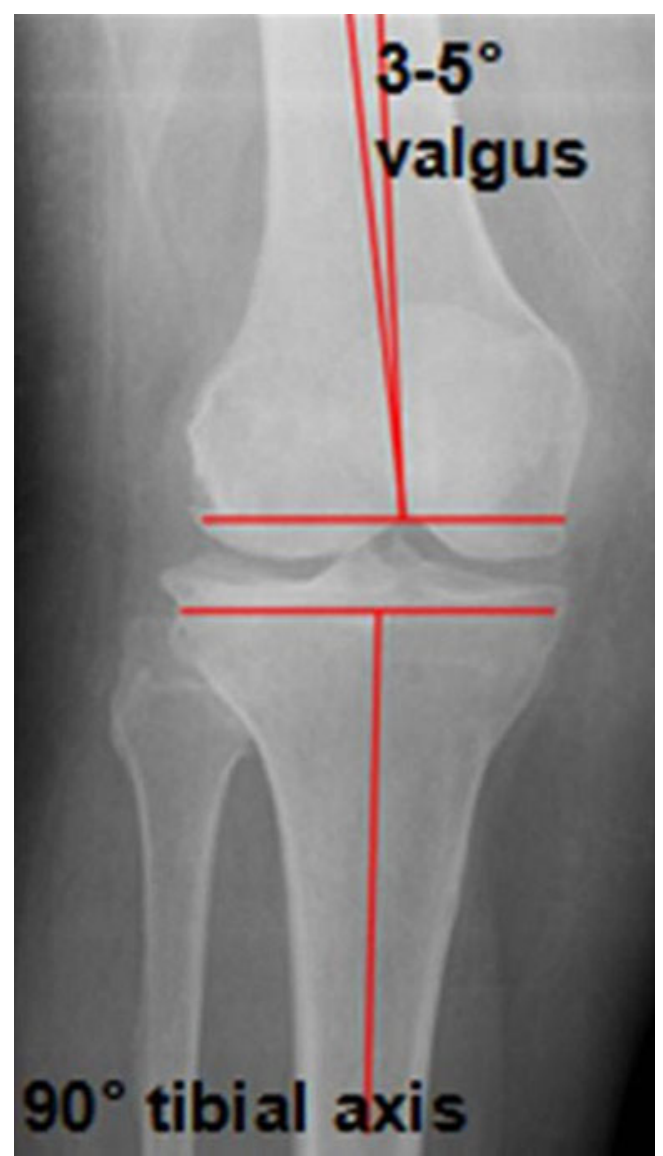

Fig. 2 Anteroposterior view of an X-ray showing our preoperative planning to evaluate the bone cuts. The femoral anatomical axis is drawn and then a second line with the desired amount of valgus (usually $3^{\circ}$ ) is also drawn at the level of the intercondylar notch
The main disadvantage of the medial approach is that it is more difficult to reach the posterolateral corner during the lateral soft tissue release. For this reason sometimes a tibial tuberosity osteotomy (TTO) is necessary; different complications have been reported with this technique. Besides, patellar vascular damage has been described when a medial parapatellar approach is combined with a lateral release [17]. Some authors reported that when a medial approach is performed in a valgus knee, the results have been inferior compared to varus deformity [18].

\section{Anterolateral approach}

In 1991 Keblish [4] described an anterolateral approach for TKA in valgus deformities. He described a long incision along the lateral border of the quadriceps muscle, taking care to leave $1 \mathrm{~cm}$ of the lateral retinaculum, from the junction between the vastus lateralis and the quadriceps tendon to the patella, through $50 \%$ of the tendon. The patella is dislocated medially. Some authors $[19,20]$ described a TTO also to protect the extensor mechanism during patellar eversion with good results [17]. According to Keblish it may be difficult to close the lateral compartment after the deformity correction. Two different tricks are described to facilitate lateral closure: (1) approximation of the infrapatellar fat pad to the patellar ligament and (2) separation of the vastus lateralis from the rectus femoris, followed by suturing together the two tendons in a staggered position [4].

According to different authors [21], the main advantage of the lateral approach is a better visualisation of the tight lateral tissues; besides, if a lateral retinaculum release is necessary,
Fig. 3 Patient with a grade II left valgus knee. a Long leg view showing the valgus deformity. b Anteroposterior view. c Lateral view showing posterior osteophytes

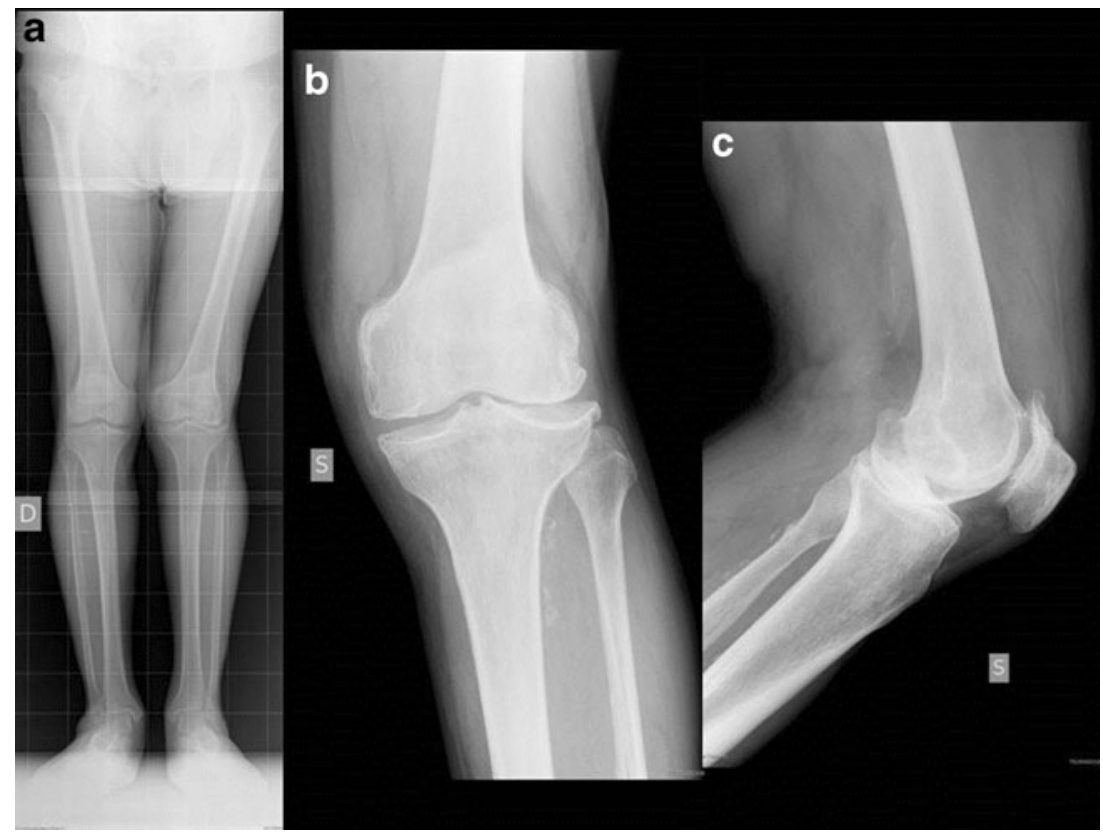


Fig. 4 Post-operative X-ray of the same patient as in Fig. 3 showing a well-positioned PS implant. a Post-operative anteroposterior and lateral views. b Anteroposterior long leg view at 3 months showing a well-aligned left knee

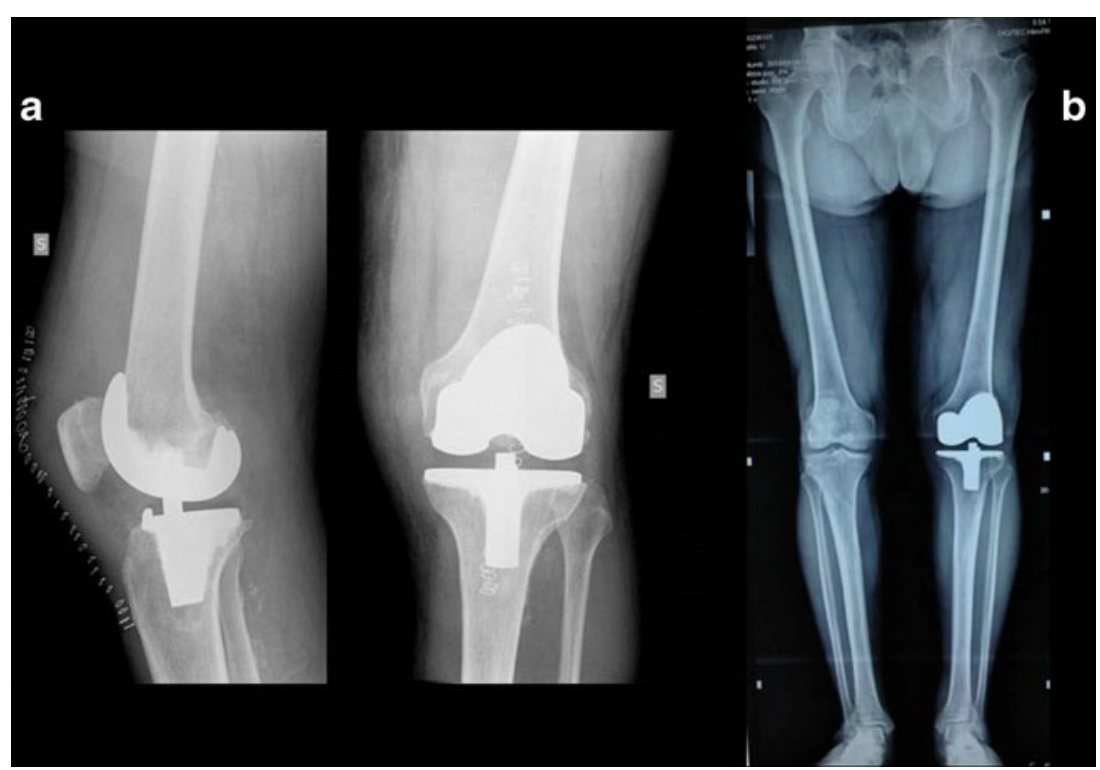

the patellar vascularisation will be not compromised. On the other hand, potential disadvantages of this technique include difficulty in patellar eversion, sometimes requiring TTO, and less familiarity of many surgeons with this technique [22].

There is an open debate about which approach leads to better results. Sekiya et al. [21] reported on clinical and radiological results in two randomised groups of patients with a valgus deformity after performing a TKA. In the first group a medial parapatellar approach was used and in the second one a lateral parapatellar approach without TTO. They found no significant differences in ROM but better post-operative flexion in the second group. Nikolopoulos et al. [23] reported on two groups: in the first a lateral parapatellar approach combined with TTO had been performed and in the second one a standard medial approach had been used. They found no statistically significant differences in terms of clinical results,
Fig. 5 Clinical examination and long leg view of the same patient as in Figs. 3 and 4 showing the association between planovalgus foot and valgus knee. a Clinical examination of the planovalgus foot. b Anteroposterior long leg view showing the valgus knee

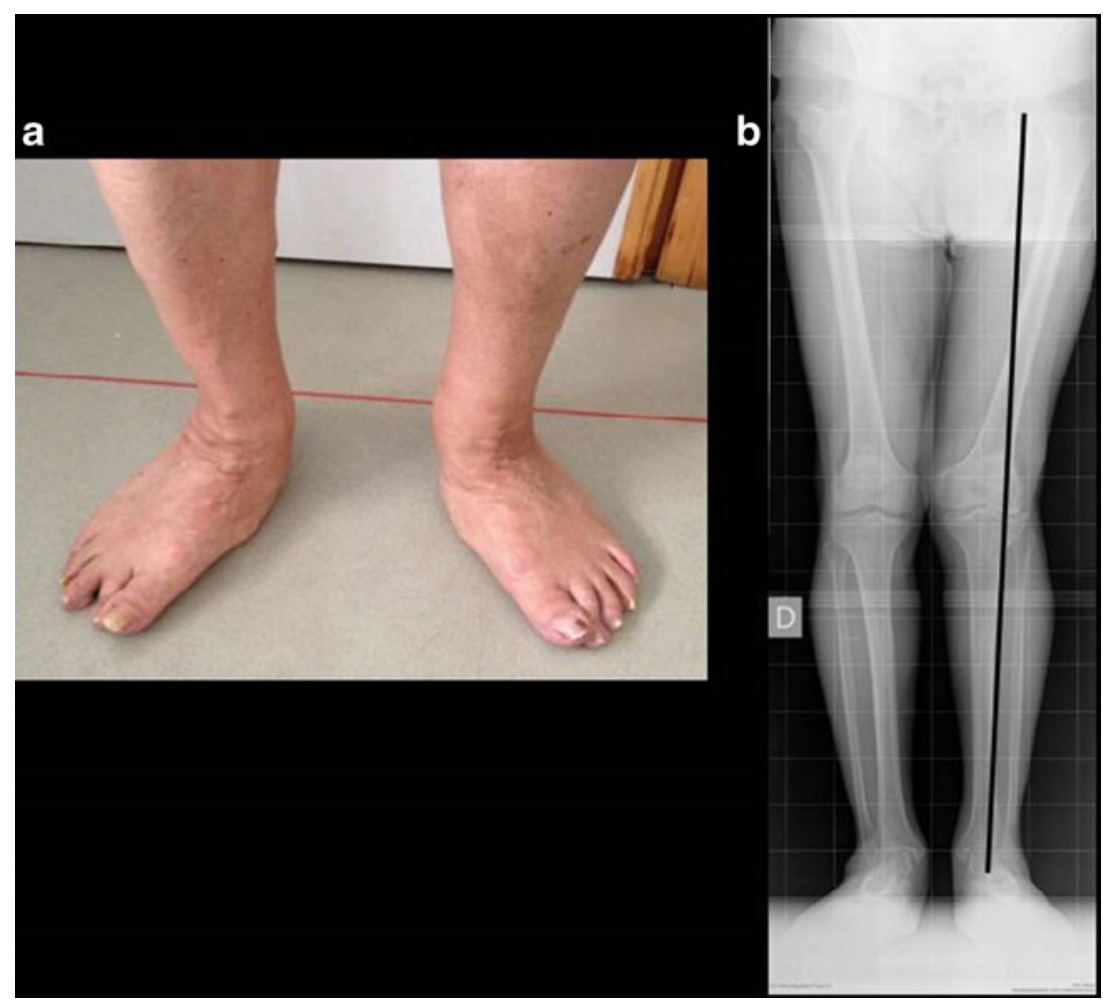


Fig. 6 Patient with a grade III bilateral valgus knee more symptomatic to the right side. a Clinical examination showing bilateral valgus knee. b, c Long leg anteroposterior X-ray view showing bilateral valgus

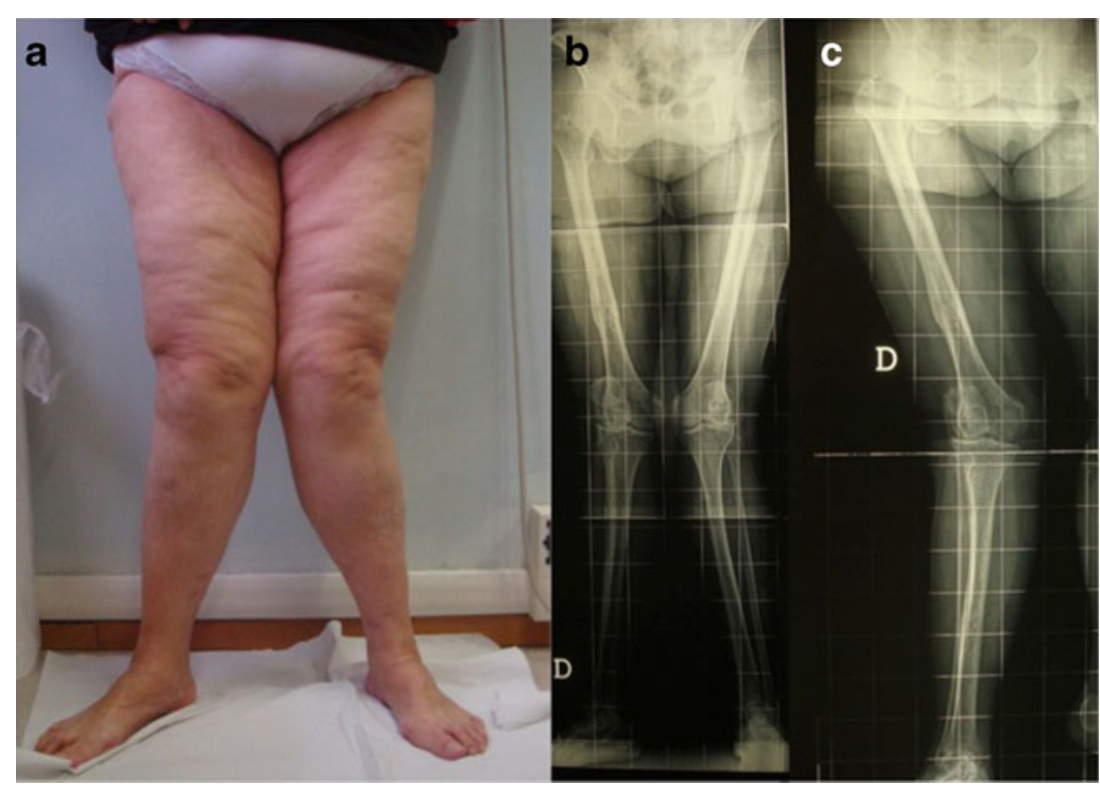

but in the lateral approach group a valgus deviation occurred in $9 \%$ of the patients, compared to $32 \%$ in the medial approach one. A similar study has been published by Hirschmann et al. [24], but they concluded that long-term studies are necessary to show whether there is any difference in prosthesis longevity between both types of approach.

\section{Bone cuts}

In valgus knee deformity bone cuts can be performed differently in order to correct low-grade deformities and reduce great deformities. In order to make the right cut the surgeon should pay attention to valgus knee bony variables: lateral
Fig. 7 Post-operative X-ray on anteroposterior and lateral views showing the rotating hinge prosthesis implanted in the same patient as in Fig. 6 . a Anteroposterior view. b Lateral view

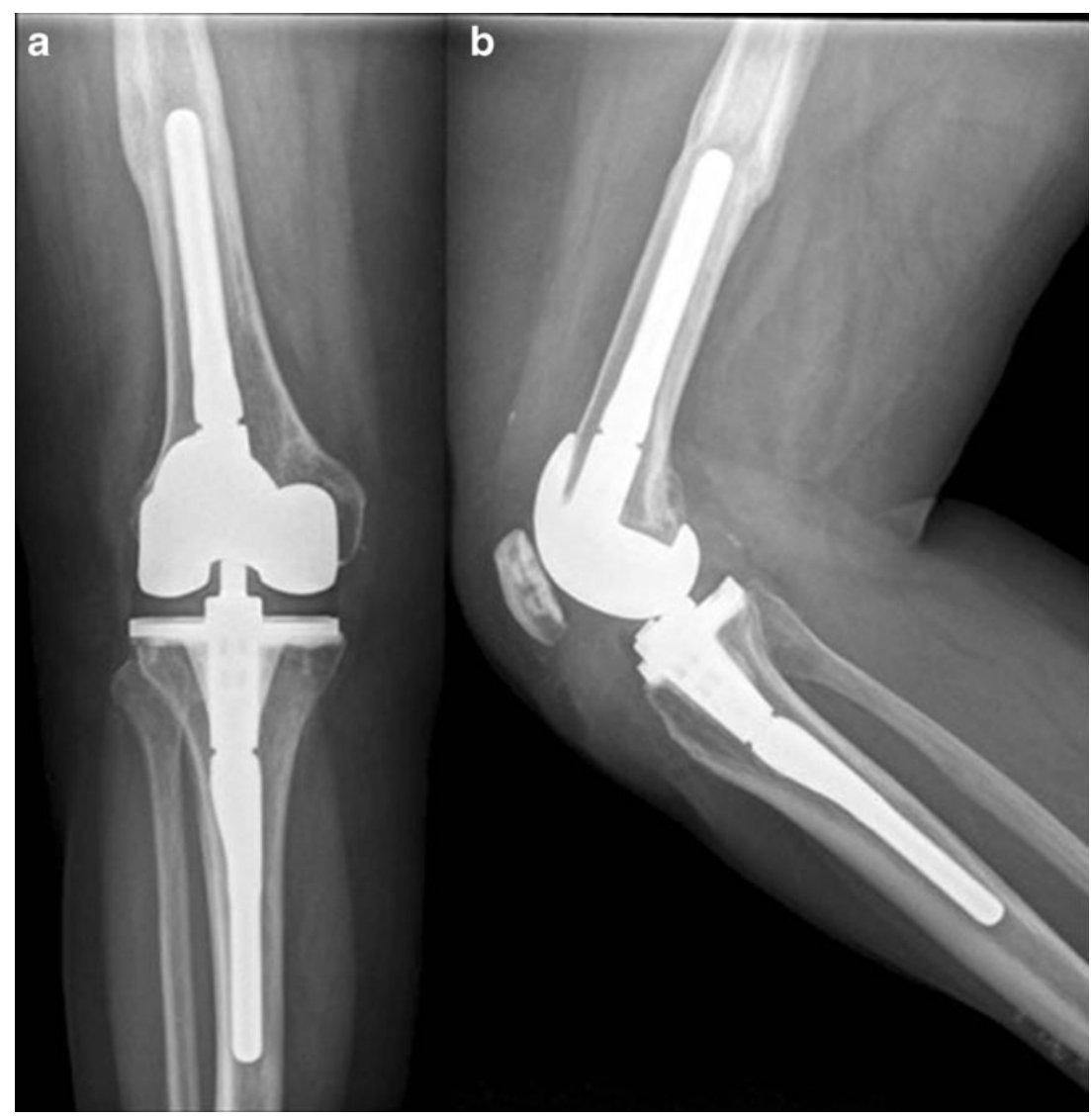


femoral condylar hypoplasia and asymmetric cartilage wear on the tibial plateau. These characteristics can influence limb alignment, component rotation and patellar tracking [1].

\section{Tibia}

The cut has to be perpendicular to the tibial long axis. The difference between these cases and a standard TKA is the amount of the resection in grade II and III valgus deformities. The resection should be from 6 to $8 \mathrm{~mm}$ in the medial compartment and always has to be performed after having removed all the osteophytes, especially in the lateral side of the tibial plateau. In cases of severe bony deformity of the tibial plateau, it can occur that almost no bone is resected on the lateral side to avoid medial over-resection or malaligned cuts.

\section{Femur}

Distal cut: It is useful for the femoral cut to reduce valgus degrees of resection from $5-7^{\circ}$ to $3^{\circ}$ in order to properly correct pre-operative deformity. Also at this level lateral condyle distal femoral resection can be minimal (1-2 mm) or absent in severe valgus deformity of the distal femur. Femoral resection should be no more than $10 \mathrm{~mm}$ in the medial condyle (usually 7-8 mm).

Anteroposterior cuts: The surgeon has to pay attention to lateral condylar hypoplasia that can determine a great intra-rotation of the components if a posterior reference is used. Considering this aspect, Arima et al. support the utility of using the anteroposterior axis in order to give the proper femoral rotation in valgus anatomy [25]. In cases of severe trochlear dysplasia, the Whiteside line can be extremely difficult to identify: in these cases the epicondylar axis or parallel to the tibial cut technique should be used to assess a correct femoral rotation.

\section{Soft tissue management}

(a) Lateral soft tissue

In the valgus knee many lateral structures are retracted: the ITB, the PLC, the LCL, the POP and the LHG.

In knees with severe valgus deformity, the PCL may also be retracted, and it cannot be retained if the surgeon wants to achieve full correction of the deformity.

There is agreement in the literature that lateral structure release is necessary in valgus deformity, but there is an open debate on which is the best sequence and the best technique to perform those releases.

The releases should be performed with the knee in extension and using lamina spreaders to check the tension of the medial and lateral compartments. After each release the surgeon should evaluate the alignment and the stability of the knee, in order to achieve a symmetrical rectangular extension and flexion gaps with the spacer block in situ.

Krackow et al. [5] normally release the ITB and LCL first in the type I valgus knee, followed by the POP and the PLC, when necessary.

Ranawat et al. [1] described a stepwise technique in which the first structure to be released is the PCL. Then they perform a PLC intra-articular release using an electrocautery at the level of the tibial cut surface. The ITB is released when necessary with multiple inside-out stab incisions, as well as the LCL; on the contrary, the POP is normally preserved.

Favorito et al. [2] described that more commonly the LCL is the tightest structure, so it is the first structure to be released. The next release is the POP, followed by the PLC, the femoral insertion of the LHG and, finally, the ITB can be considered.

Different authors tried to described a lateral structure release sequence based on functional and anatomical consideratios. Most of them agree in describing the POP as an important structure for rotational and valgus stability in flexion.

Whiteside [26] described a soft tissue release sequence based on the functional effect of the single structures. In his opinion, a ligament attached to the femur near the epicondyles, so near the axis through which the tibia rotates and the knee flexes and extends, has an important role in flexion stability. On the other hand, a ligament attached far away from the epicondyle is more important for the extension knee stability. Following this theory, the LCL and POP, which are attached to the epicondyle, are important lateral stabilisers in flexion; these two structures are appropriate to release for a knee that is tight in flexion. On the contrary, the ITB and the PLC are important knee stabilisers in extension, so they should be released when the knee is tighter in extension. Whiteside concluded that a standard lateral release sequence is not applicable to all patients, but the surgeon should release a different structure if the knee is tight in flexion, in extension or during all ROM.

Krackow and Mihalko [27] published a cadaveric study in which they studied the amount of correction achieved with each release step of two different sequences, comparing it in flexion and extension and measuring any rotational changes of the tibia. The sequences were: ITB, POP, LCL and LHG and LCL, POP, ITB and LHG. They evaluated the amount of correction at 0,45 and $90^{\circ}$ of flexion. The results showed that the greatest varus rotation occurred once all structures were released, with the LHG origin last in 


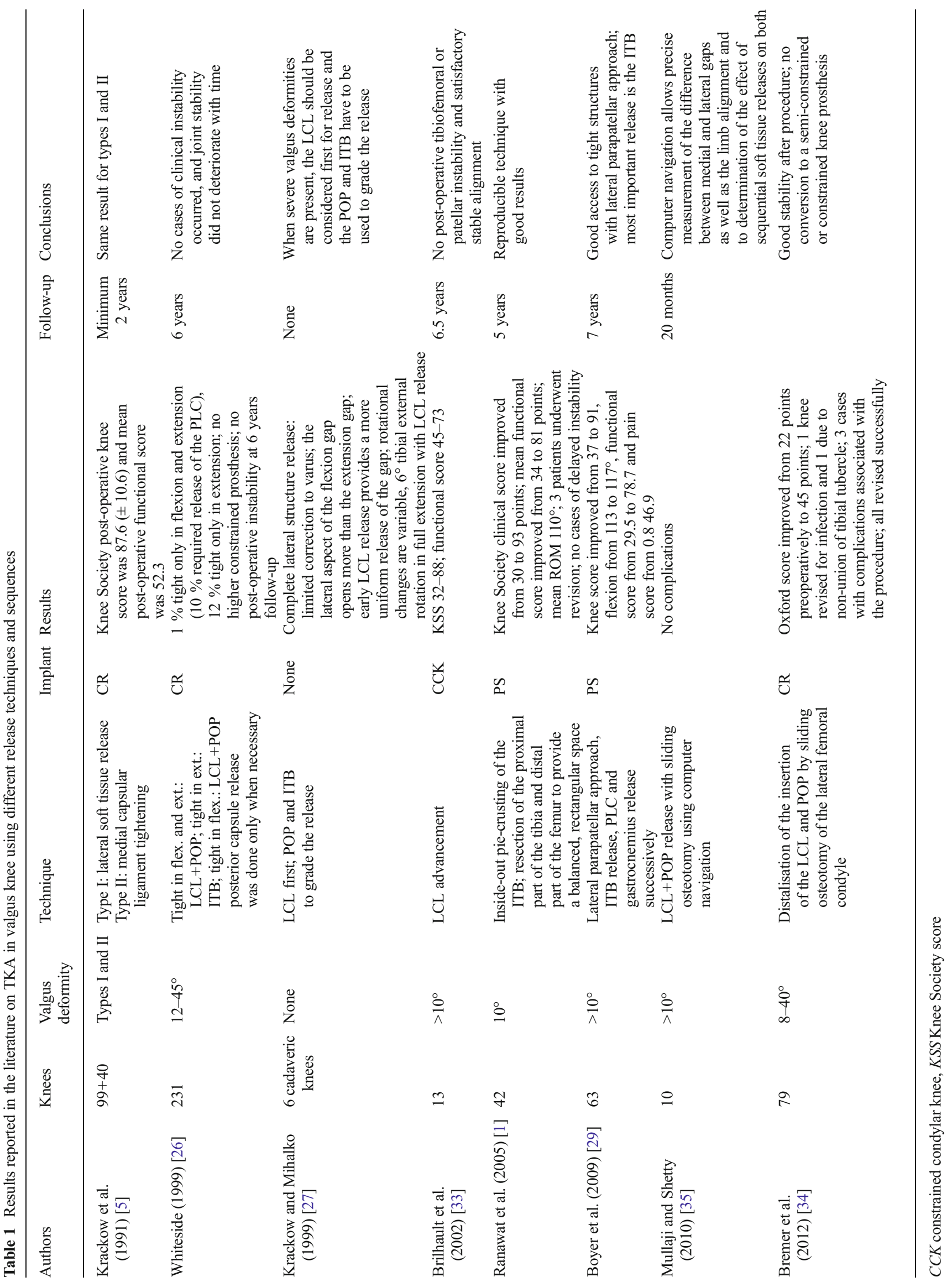


both groups. Moreover, the largest increase occurred after the release of the LCL. They concluded that in severe valgus deformities, the LCL should be considered first for release; POP and ITB should be used to grade the release.

Three main techniques are described in the literature to perform lateral soft tissue release.

Different authors described a subperiosteal technique or a Z-plasty technique to perform the lateral releases [26-28]. In the technique described by Krackow and Mihalko [27] the knee was fully extended and the ligaments were palpated to ascertain which structures were tightest. Typically the ITB and the LCL were released first with a subperiosteal technique, followed by the POP and occasionally by the PLC. The LHG was released only if a flexion contracture were present. In the technique described by Whiteside [26] the LCL and the POP were released directly from the bone attachments leaving the periosteum intact. The ITB was released subcutaneously and released extrasynovially.

Other authors [4, 29] used a lateral parapatellar approach and automatically released the ITB from Gerdy's tubercle.

Finally, Ranawat's "pie-crusting technique" has been described for releasing the lateral structure with an inside-out procedure. In this technique the tight lateral structures are palpated when the lamina spreaders are inserted, and they are released performing multiple transverse incisions with a no. 15 surgical blade [30, 31]. One of the disadvantages of this technique is the potential risk of peroneal nerve lesion. Bruzzone et al. [32], in a cadaveric study, concluded that the nerve is at overall risk during the release of the PLC, in the triangle defined by the POP, the tibial cut surface and the most posterior fibres of the ITB ("danger zone"), but not during the pie-crusting of the ITB ("safe zone").

An alternative technique for lateral structure release has been described by Brilhault et al. associated with a lateral parapatellar approach [33]. A sliding osteotomy of the femoral LCL and POP insertions is done and the resulting bone block is mobilised and placed more distally. This procedure produces a rectangular space and had great results in Bremer et al.'s study: no conversion to semi-constrained or constrained knee prosthesis [34]. Mullaji and Shetty [35] described a similar technique in which, after the release of the PLC and the ITB, they performed a computer navigated lateral epicondylar osteotomy, with a more accurate repositioning of the epicondyle.

(b) Medial soft tissue

As described by Krackow et al. [5] in grade II valgus deformities the MCL may not be completely functional, and a residual medial laxity is poorly tolerated if a valgus deformity persists post-operatively. In these conditions the authors suggested tightening the medial ligamentous structures, particularly if the PCL has been retained. In 1990 Krackow et al. [36] described a tightening technique in which a small bone plug with the attached insertion of the PCL and the PLC is removed from the tibia and moved distally, securing it with transosseous sutures. In this technique the MCL is tightened by moving a bone block distally with its tibial insertion. Other techniques have been described. The advancement of the MCL from the epicondyle or a division and imbrication in order to tighten it can be performed in conjunction with the use of a constrained condylar prosthesis [2].

\section{Complications}

In the literature different main complications have been described [2]:

- $\quad$ Tibiofemoral instability (2-70\%)

- Recurrent valgus deformity (4-38\%)

- Poor post-operative ROM (1-20\%)

- Wound problems (4-13\%)

- Patellar stress fracture and osteonecrosis (1-12\%)

- Patellar maltracking (2-10\%)

- Peroneal nerve palsy $(0.3-9.5 \%)[37,38]$

Correction of a valgus deformity places the peroneal nerve at risk for indirect injury via traction, ischaemia or both. Apart from indirect mechanisms, concern has been expressed over the use of the Ranawat inside-out release, which may place the nerve at risk for direct laceration-type injuries. Direct injury to the peroneal nerve is most likely to occur during the posterolateral release of the capsule carried out at the level of the tibial cut using the Ranawat technique than during ITB pie-crusting [32].

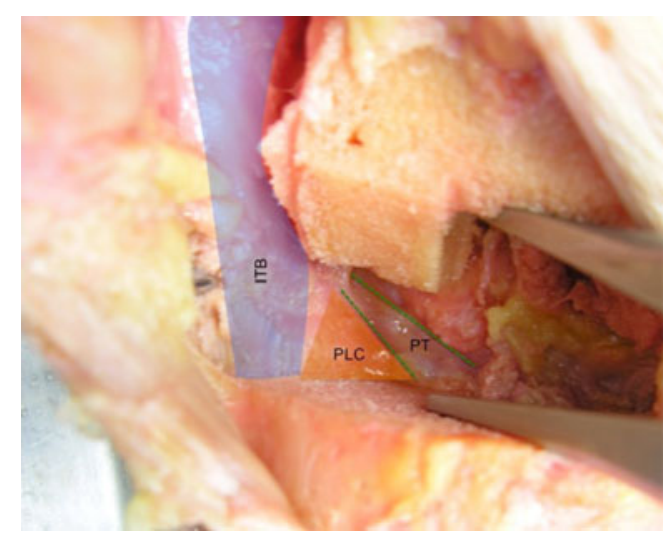

Fig. 8 Cadaveric anatomy showing the POP, PLC and ITB 


\section{Results}

Table 1 summarises the results in the literature with different release sequences.

\section{Our technique}

In all cases we perform weight-bearing anteroposterior, lateral, Rosenberg, Merchant and long leg views to assess the lower limb alignment and the distal femoral and proximal tibial remodelling. We perform pre-operative planning using the technique we described in the previous section (Fig. 2).

We routinely use a PS implant in type I deformity. In type II deformity we decide the level of constraint on the operative field, based on the integrity and functionality of the MCL. If there is a medial residual instability we do not perform a medial tightening, but we prefer to switch to a higher constrained implant. In type III deformities we routinely use a condylar constrained or, in the most severe cases, a rotating hinge prosthesis.

Antibiotic prophylaxis is routinely administered to the patients (depending on the hospital's protocols), and they are positioned supine, as in a routine implant.

We routinely perform a medial parapatellar approach, being really careful not to excessively release medial structures, minimising medial dissection to fully visualise the tibia. We first perform the tibial cut, perpendicular to the anatomical axis, removing the smallest possible bone amount, especially from the lateral side. For the femoral cuts we reduce the valgus degree of resection to $3^{\circ}$, in order to avoid under-correction of the deformity. We pay attention to correctly access the femoral canal with the intramedullary rod: the entry point in a valgus knee is usually more medial than in a standard knee. Pre-operative radiographs are extremely useful to correctly access the canal avoiding gross mistakes.

With regard to anteroposterior cuts, we take extreme care in evaluating possible hypoplasia of the lateral femoral condyle and we triple-check the posterior condylar reference cutting block position with both Whiteside line and transepicondylar axis; moreover, with the cutting blocks in site, we further check the balancing in flexion before performing the cuts.

We follow the "three-step technique" described by Ranawat et al. to evaluate the lateral soft tissue release [1]. When distal femoral and proximal tibial cuts are performed, the osteophytes have been removed and the PCL has been sacrificed, we put the knee in extension with lamina spreaders to evaluate the extension gap. At this point we routinely perform a selective lateral soft tissue release. We palpate the lateral structure and we proceed with a pie-crusting release just of the structures that are really tight, starting from the PLC, followed by the ITB. We normally preserve the POP because of its importance in flexion stability. Figure 8 shows the anatomy of the lateral structures. The knee is then stressed in varus/valgus to evaluate the stability in extension and, if further releases are necessary, we restart the procedure. In accordance with Ranawat et al. [1] we try to preserve at least one or two lateral stabilisers. If instability is detected after the releases have been performed we switch to a condylar constrained prosthesis. Once the knee is balanced in extension, the flexion gap can be evaluated and assessed. When the extension and flexion gaps are equal, femoral chamfer cuts can be made and the trial components can be tested. To assess tibial component rotation and sizing, we use the posterolateral corner locked technique we previously described [39].

At this point it is important to evaluate patellar tracking with the tourniquet deflated and the "no thumb" technique. If the trial components are well positioned and a lateral release is still necessary, a pie-crusting release of the lateral retinaculum can be performed. At this point the knee is irrigated, the tourniquet is inflated and the components are cemented in position. During the pressurisation cement excess is removed. After cement polymerisation, the knee is checked in all positions and a drain is positioned intra-articularly. The capsule is closed in flexion taking care to avoid patella baja or alta.

The post-operative protocol is standard with continuous passive motion from day one and weight-bearing with crutches as tolerated from day two. Controls are clinical after two months, radiographic and clinical at three months and one year and successively every two years.

Conflict of interest The authors declare that they have no conflict of interest.

\section{References}

1. Ranawat AS, Ranawat CS, Elkus M, Rasquinha VJ, Rossi R, Babhulkar S (2005) Total knee arthroplasty for severe valgus deformity. J Bone Joint Surg Am 87 Suppl 1(Pt 2):271-284

2. Favorito PJ, Mihalko WM, Krackow KA (2002) Total knee arthroplasty in the valgus knee. J Am Acad Orthop Surg 10(1):16-24

3. Ritter MA, Davis KE, Davis P, Farris A, Malinzak RA, Berend ME, Meding JB (2013) Preoperative malalignment increases risk of failure after total knee arthroplasty. J Bone Joint Surg Am 95(2):126-131

4. Keblish PA (1991) The lateral approach to the valgus knee. Surgical technique and analysis of 53 cases with over two-year follow-up evaluation. Clin Orthop Relat Res 271:52-62

5. Krackow KA, Jones MM, Teeny SM, Hungerford DS (1991) Primary total knee arthroplasty in patients with fixed valgus deformity. Clin Orthop Relat Res 273:9-18

6. Robbins GM, Masri BA, Garbuz DS, Duncan CP (2001) Preoperative planning to prevent instability in total knee arthroplasty. Orthop Clin North Am 32(4):611-626

7. Krackow KA (1990) The technique of total knee arthroplasty. Mosby, St. Louis 
8. Matsuda Y, Ishii Y, Noguchi H, Ishii R (2005) Varus-valgus balance and range of movement after total knee arthroplasty. J Bone Joint Surg Br 87:804-808

9. Mihalko WM, Krackow KA (2000) Anatomic and biomechanical aspects of pie crusting posterolateral structures for valgus deformity correction in total knee arthroplasty: a cadaveric study. J Arthroplasty $15: 347-353$

10. Meding JB, Keating EM, Ritter MA, Faris PM, Berend ME, Malinzak RA (2005) The planovalgus foot: a harbinger of failure of posterior cruciate-retaining total knee replacement. J Bone Joint Surg Am 87(Suppl 2):59-62

11. Pang HN, Yeo SJ, Chong HC, Chin PL, Chia SL, Lo NN (2013) Joint line changes and outcomes in constrained versus unconstrained total knee arthroplasty for the type II valgus knee. Knee Surg Sports Traumatol Arthrosc 21(10):2363-2369

12. Dettoni F, Bonasia DE, Castoldi F, Bruzzone M, Blonna D, Rossi R (2010) High tibial osteotomy versus unicompartmental knee arthroplasty for medial compartment arthrosis of the knee: a review of the literature. Iowa Orthop J 30:131-140

13. Windsor RE, Insall JN, Vince KG (1988) Technical considerations of total knee arthroplasty after proximal tibial osteotomy. J Bone Joint Surg Am 70:547-555

14. Nelson CL, Saleh KJ, Kassim RA, Windsor R, Haas S, Laskin R, Sculco T (2003) Total knee arthroplasty after varus osteotomy of the distal part of the femur. J Bone Joint Surg Am 85-A(6):1062-1065

15. Rajgopal A, Vasdev A, Dahiya V, Tyagi VC, Gupta H (2013) Total knee arthroplasty in extra articular deformities: a series of 36 knees. Indian J Orthop 47(1):35-39

16. Xiao-Gang Z, Shahzad K, Li C (2012) One-stage total knee arthroplasty for patients with osteoarthritis of the knee and extraarticular deformity. Int Orthop 36(12):2457-2463

17. Buechel FF (1990) A sequential three-step lateral release for correcting fixed valgus knee deformities during total knee arthroplasty. Clin Orthop Relat Res 260:170-175

18. Apostolopoulos AP, Nikolopoulos DD, Polyzois I, Nakos A, Liarokapis S, Stefanakis G et al (2010) Total knee arthroplasty in severe valgus deformity: interest of combining a lateral approach with a tibial tubercle osteotomy. Orthop Traumatol Surg Res 96(7): 777-784

19. Whiteside LA (1993) Correction of ligament and bone defects in total arthroplasty of the severely valgus knee. Clin Orthop 288:234-245

20. Wolff AMN, Hungerford DS, Pepe CL (1991) The effect of extraarticular varus and valgus deformity on total knee arthroplasty. Clin Orthop 271:35-51

21. Sekiya H, Takatoku K, Takada H, Sugimoto N, Hoshino Y (2012) Lateral approach is advantageous in total knee arthroplasty for valgus deformed knee. Eur J Orthop Surg Traumatol

22. Fiddian NJ, Blakeway C, Kumar A (1998) Replacement arthroplasty of the valgus knee. A modified lateral capsular approach with repositioning of vastus lateralis. J Bone Joint Surg Br 80:859-861

23. Nikolopoulos DD, Polyzois I, Apostolopoulos AP, Rossas C, Moutsios-Rentzos A, Michos IV (2011) Total knee arthroplasty in severe valgus knee deformity: comparison of a standard medial parapatellar approach combined with tibial tubercle osteotomy. Knee Surg Sports Traumatol Arthrosc 19(11):1834-1842

24. Hirschmann MT, Hoffmann M, Krause R, Jenabzadeh RA, Arnold MP, Friederich NF (2010) Anterolateral approach with tibial tubercle osteotomy versus standard medial approach for primary total knee arthroplasty: does it matter? BMC Musculoskelet Disord 11:167

25. Arima J, Whiteside LA, McCarthy DS, White SE (1995) Femoral rotational alignment, based on the anteroposterior axis, in total knee arthroplasty in a valgus knee. A technical note. J Bone Joint Surg Am 77(9):1331-1334

26. Whiteside LA (1999) Selective ligament release in total knee arthroplasty of the knee in valgus. Clin Orthop Relat Res 367: $130-140$

27. Krackow KA, Mihalko WM (1999) Flexion-extension joint gap changes after lateral structure release for valgus deformity correction in total knee arthroplasty: a cadaveric study. J Arthroplasty 14(8): 994-1004

28. Engh GA (2003) The difficult knee: severe varus and valgus. Clin Orthop Relat Res 416:58-63

29. Boyer P, Boublil D, Magrino B, Massin P, Huten D, Guepar Group (2009) Total knee replacement in the fixed valgus deformity using a lateral approach: role of the automatic iliotibial band release for a successful balancing. Int Orthop 33(6): 1577-1583

30. Aglietti P, Lup D, Cuomo P, Baldini A, De Luca L (2007) Total knee arthroplasty using a pie-crusting technique for valgus deformity. Clin Orthop Relat Res 464:73-77

31. Clarke HD, Fuchs R, Scuderi GR, Scott WN, Insall JN (2005) Clinical results in valgus total knee arthroplasty with the "pie crust" technique of lateral soft tissue releases. J Arthroplasty 20(8): $1010-1014$

32. Bruzzone M, Ranawat A, Castoldi F, Dettoni F, Rossi P, Rossi R (2010) The risk of direct peroneal nerve injury using the Ranawat "inside-out" lateral release technique in valgus total knee arthroplasty. J Arthroplasty 25(1):161-165

33. Brilhault J, Lautman S, Favard L, Burdin P (2002) Lateral femoral sliding osteotomy lateral release in total knee arthroplasty for a fixed valgus deformity. J Bone Joint Surg Br 84(8):1131-1137

34. Bremer D, Orth BC, Fitzek JG, Knutsen A (2012) Briard's sagittal sliding osteotomy of the lateral condyle in total knee arthroplasty of the severe valgus knee. Oper Orthop Traumatol 24(2):95-108

35. Mullaji AB, Shetty GM (2010) Lateral epicondylar osteotomy using computer navigation in total knee arthroplasty for rigid valgus deformities. J Arthroplasty 25(1):166-169

36. Krackow KA, Holtgrewe JL (1990) Experience with a new technique for managing severely overcorrected valgus high tibial osteotomy at total knee arthroplasty. Clin Orthop Relat Res 258:213-224

37. Nercessian OA, Ugwonali OF, Park S (2005) Peroneal nerve palsy after total knee arthroplasty. J Arthroplasty 20:1068-1073

38. Schinsky MF, Macaulay W, Parks ML et al (2001) Nerve injury after primary total knee arthroplasty. J Arthroplasty 16:1048-1054

39. Rossi R, Bruzzone M, Bonasia DE, Marmotti A, Castoldi F (2010) Evaluation of tibial rotational alignment in total knee arthroplasty: a cadaver study. Knee Surg Sports Traumatol Arthrosc 18(7):889-893 